\title{
Association of Meat Consumption With NAFLD Risk and Liver-related Biomarkers in Older Chinese: A Cross-sectional Study
}

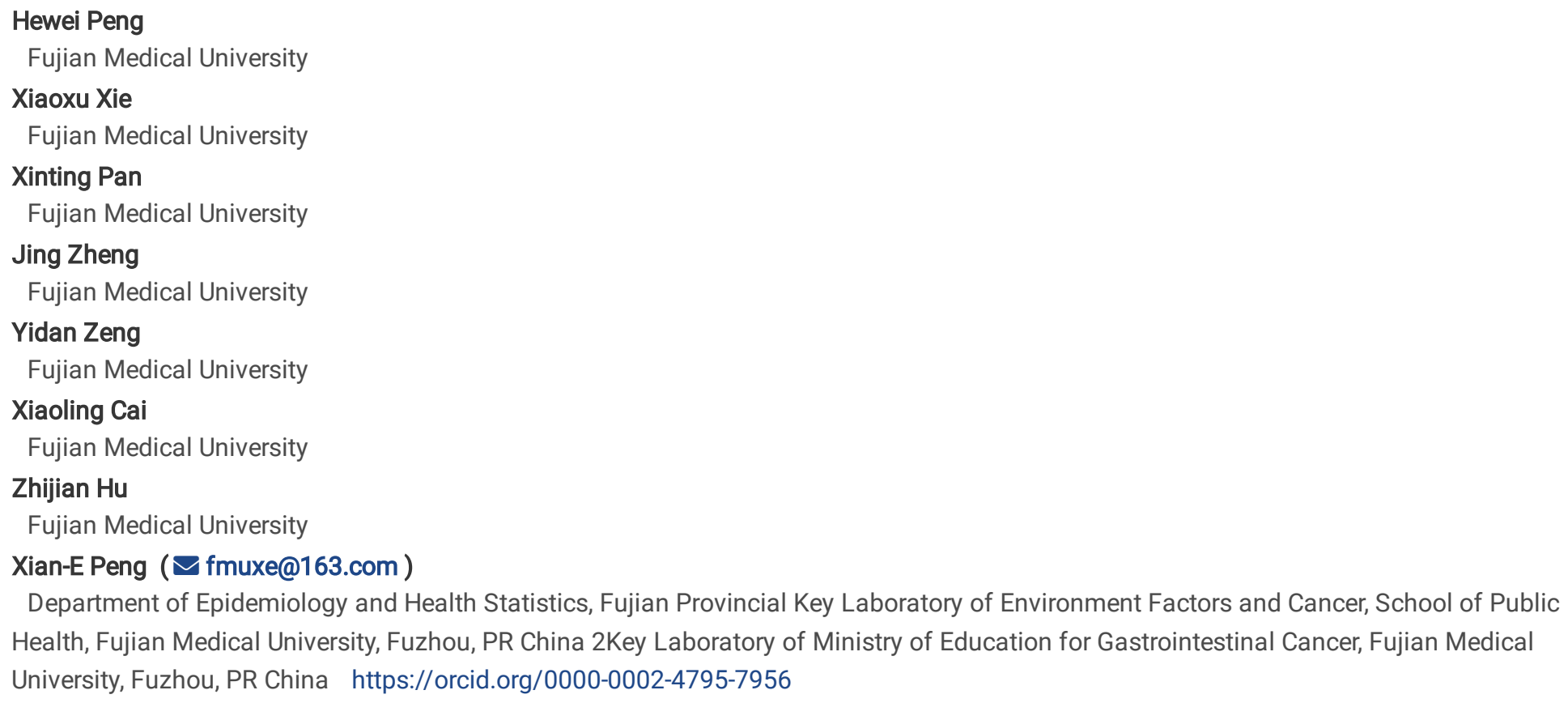

Research article

Keywords: non-alcoholic fatty liver disease, meat, liver biomarkers

Posted Date: September 8th, 2020

DOI: https://doi.org/10.21203/rs.3.rs-63427/v1

License: @ (1) This work is licensed under a Creative Commons Attribution 4.0 International License. Read Full License 


\section{Abstract}

Background: High meats intake contributes to unhealthy status. The present study aims to evaluate the association of meat consumption with non-alcohol fatty liver disease (NAFLD) risk and liver-related biomarkers in middle-aged and elderly Chinese.

Methods: A cross-sectional study was conducted in individuals who were 45 years or older and underwent physical examination from April 2015 to August 2017 in Southeast China. NAFLD was evaluated by abdominal ultrasonography.

Results: High consumptions of red meat (28.44-49.74 and $>71.00 \mathrm{~g} /$ day) was positively associated with NAFLD risk on inverse probability of treatment weighting analysis, adjusting for smoking, tea intake, weekly hours of physical activity and presence of hypertension, dyslipidemia and diabetes $\left(O R_{\text {adjusted }}=1.948\right.$ and 1.1.716, respectively). Exposure-response relationship analysis presented that red meat intake was positively associated with NAFLD risk. Significant associations of red meat intakes with serum levels of $y$-glutamyl transferase, alanine transaminase, aspartate aminotransferase, total triglyceride and high-density lipoprotein cholesterol were found $\left(r_{\mathrm{s}}=0.176,0.128\right.$, $0.060,0.085$ and -0.074 , respectively).

Conclusions: These findings suggest that reduction of meat consumption may decrease NAFLD risk and should warrant further investigations. Meat consumptions were measured by a semi-quantitative food frequency questionnaire.

\section{Background}

Non-alcoholic fatty liver disease (NAFLD) is the most common liver disease associated with the metabolic syndrome or its components and is becoming a major global health and economic burden, with a $25 \%$ prevalence worldwide [1]. It is defined as the presence of more than $5 \%$ of hepatic steatosis, with little or no secondary causes of fatty liver, such as alcohol, virus and drugs. In addition, NAFLD is associated not only with adverse hepatic outcomes included cirrhosis and liver cancer, but also with non-liver-associated adverse outcomes, such as cardiovascular diseases and diabetes [2-4]. These data highlight a serious concern for the future, and the enormous increasing health burden of NAFLD.

Lifestyle including dietary habits positively influenced the development and progression of NAFLD [5, 6]. Unhealthy dietary patterns including high intake of soft drink and of meat have been demonstrated to be significantly increased the NAFLD risk [7-10]. Meat is an important source of energy and some indispensable nutrients, including proteins and some micro-nutrients such as iron, zinc, selenium and B-vitamins [11, 12]. However, an increased consumption of meat contributes to high intakes of dietary cholesterol, saturated fat and other harmful compounds, which are closely connected to the NAFLD [13]. The global average per capita consumption of meat is rising, especially in China [11]. Chinese meat consumption increased from $58.9 \mathrm{~g} / \mathrm{d}$ to $89.7 \mathrm{~g} / \mathrm{d}$ during 1992 to 2012, which was far beyond the recommendation for Chinese adults [13]. Moreover, high consumption of meat were related to insulin resistance, type 2 diabetes, lipodystrophy, cardiovascular diseases, hepatocellular carcinoma and hypertension[14-17], which coexist and share similar pathogenesis of NAFLD $[18,19]$. Therefore, it is important to examine the relationship of meat intake with NAFLD among Chinese population, especially in middle-aged and elders, who are predisposed to NAFLD [20].

Furthermore, liver-related clinical parameters such as y-glutamyl transferase (GGT), aspartate aminotransferase (AST) and alanine transaminase (ALT) were closely associated with NAFLD [21]. Elevated GGT, AST ALT and were the markers underlying liver diseases such as NAFLD [22]. However, epidemiological studies regarding the associations of meat subtypes intake with NAFLD risk and liver-related biochemical indexes were not fully addressed.

This cross-sectional study, therefore, aims to evaluate the associations of meat subtypes intake with the risk of NAFLD and related biomarkers among middle-aged and elder Chinese population.

\section{Methods}

\section{Study Subjects and Design}

This was a cross-sectional study, conducted in the health examination center of the Affiliated Nanping First Hospital, Fujian Medical University from April 2015 through August 2017. We included individuals aged $\geq 45$ years old and who permanently resided in Nanping. Further exclusion criteria in our study were as follows: (A) Individuals whose daily consumptions of alcohol $>40 \mathrm{~g}$ (men) or $>20 \mathrm{~g}$ (women). (B) Individuals who had any other liver diseases history, such as drug-induced liver disease, viral hepatitis, Wilson's disease, autoimmune hepatitis and total parenteral nutrition. (C) Individuals who were taking hypolipidemic or diet pills. (D) Individuals who did not answer more 
than 25 food-related items on the questionnaire. (E) Individuals who did not provide information on smoking and tea consumption. All subjects provided their informed consent prior to participating in this study.

The current study was carried out in compliance with the Declaration of Helsinki, and the Ethics Committee of Fujian Medical University approved the study protocol (ethics number 2014096).

\section{Data Collection}

\section{NAFLD ascertainment}

NAFLD was diagnosed by abdominal ultrasonography using established criteria [23]. Abdominal ultrasonography examination was done by experienced radiologists who were unaware of the laboratory and clinical data.

\section{Meat intakes assessment}

Semi-quantitative food frequency questionnaire included meat consumption were obtained from participants who were interviewed face to face by trained investigators. For each food item, participants used the following response options to indicate how often they ate the selected food on average: 3 times/day, twice/day, once/day, 5-6 times/week, 3-4 times/week, 1-2 times/week, 1-3 times/month, <once/month and rarely. Red meat consisted of pork, beef and lamb. Poultry was composed of chicken and duck. Processed meat contained fried and smoked meat. Fish included: fish, shellfish and crab.

\section{Covariate assessment}

The following variables were self-reported: age, sex, marital status, income, educational level, smoking status, tea intake status, physical activity, medication use and medical conditions. All subjects underwent physical examinations (height, weight, waistline, hipline and blood pressure) and blood tests (fasting plasma-glucose, low-density lipoprotein cholesterol, total cholesterol, TG, HDL-C, AST, ALT and GGT) performed by trained physicians. BMI was calculated as weight/ (height) ${ }^{2}$. Participants with a systolic blood pressure $\geq 140$ mmHg or diastolic blood pressure $\geq 90 \mathrm{mmHg}$ were defined as having hypertension. And subjects who had one or more of the following abnormalities were diagnosed as dyslipidemia: total cholesterol $\geq 6.2 \mathrm{mmol} / \mathrm{L}, \mathrm{TG}>2.25 \mathrm{mmol} / \mathrm{L}$, low-density lipoprotein cholesterol $>4.13 \mathrm{mmol} / \mathrm{L}$ or HDL-C $<1.03 \mathrm{mmol} / \mathrm{L}$ [24]. Diabetes was diagnosed as follows: fasting plasma glucose of $7.0 \mathrm{mmol} / \mathrm{L}$ or greater or 2-hour postprandial glucose greater than or equal to $11.1 \mathrm{mmol} / \mathrm{L}$.

\section{Statistical Analysis}

Nonparametric Kruskal-Wallis test and Chi-Square test were performed for comparisons between groups. Continuous variables were expressed as median (interquartile range, $I Q R$ ). Propensity scores were used to explain potential confounders by observed characteristics at baseline [25]. Age, gender and BMI were used to calculate propensity score. Inverse probability of treatment weighting was performed to evaluate the associations of red meat, processed meat, poultry and fish intakes with NAFLD, adjusting for smoking status, tea intake status, weekly hours of physical activity, and presence of hypertension, dyslipidemia and diabetes. The lowest quartile $\left(Q_{1}\right)$ of each type of meat intake was served as the reference group and $P$ for trend was calculated by set the meat intake quartiles as continuous variable. To evaluate the dose-response relationships between continuous exposure variables (red meat, processed meat, poultry and fish intakes) and NAFLD, a logistic model with restricted cubic spline using five knots at 0.05, 0.275, 0.5, 0.725 and 0.95 was built, adjusting for age, sex, BMI, smoking status, tea intake status, weekly hours of physical activity, and presence of hypertension, dyslipidemia and diabetes.

We also performed subgroup analysis to examine the relationships of red meat, processed meat, poultry and fish with NAFLD by the following subgroups: age (<60 years or $\geq 60$ years), gender (men or women), BMI ( $<24 \mathrm{~kg} / \mathrm{m}^{2}$ or $\geq 24 \mathrm{~kg} / \mathrm{m}^{2}$ ), smoking status (never, former or current), tea consumption status (yes or no), hypertension (yes or no), dyslipidemia (yes or no), diabetes (yes or no), and weekly hours of physical activity (<9 hours/week or $\geq 9$ hours/week). $P$ value for interaction was calculated. Two sensitivity analyses were conducted: (1) logistic regression analysis without IPTW; and (2) propensity score-matching logistic regressions. Furthermore, to investigate the associations of meat subtypes intakes with the concentrations of serum GGT, ALT, AST, fasting plasma glucose, total cholesterol, TG, lowdensity lipoprotein cholesterol and HDL-C, spearman's rank correlation was performed.

For the statistical analyses, SPSS, version 19.0.0.1(IBM SPSS, 2010, Chicago, IL, USA) and R, version 4.0.0 (R Project for Statistical Computing) were performed. All $P$ values were two-tailed and results were considered to be statistically significant at $P$ values $<0.05$.

\section{Results}

\section{Baseline characteristics}


A total of 1594 individuals were included in this analysis. In the entire study sample, $53.5 \%$ were men, average age was $53.54 \pm 6.90$ years and average body mass index (BMI) was $23.77 \pm 2.99 \mathrm{~kg} / \mathrm{m}^{2}$. As shown in Table 1,compared with participants with lower total intake of red, processed meat, poultry and fish, participants with higher total intake (1) were younger and more likely to be married persons, male, smokers and tea drinkers; (2) had higher educational level, higher income, higher BMI, higher waist and hip circumference; (3) had higher levels of plasma y-glutamyl transferase and alanine aminotransferase; and (4) had higher prevalence rate of NAFLD (each $P<0.05$ ).

Table 1. Baseline characteristics of the study population by quartiles of the total intake of red, processed meat, poultry and fish 


\begin{tabular}{|c|c|c|c|c|c|}
\hline & $\begin{array}{l}Q_{1}(<65.12 \\
\mathrm{g} / \text { day })\end{array}$ & $\begin{array}{l}Q_{2}(65.12-95.00 \\
\text { g/day) }\end{array}$ & $\begin{array}{l}Q_{3}(95.01-150.80 \\
\mathrm{g} / \text { day })\end{array}$ & $\begin{array}{l}Q_{4}(>150.80 \\
\mathrm{g} / \text { day })\end{array}$ & $P$ \\
\hline Age (years), $M(I Q R)$ & $53(49-59)$ & $52(49-58)$ & $52(48-57)$ & $52(48-57)$ & 0.010 \\
\hline Gender, n (\%) & & & & & $<0.001$ \\
\hline Male & $129(39.7)$ & $223(47.2)$ & $237(59.4)$ & $263(66.1)$ & \\
\hline Female & $196(60.3)$ & $249(52.8)$ & $162(40.6)$ & 135(33.9) & \\
\hline NAFLD, n (\%) & $84(25.8)$ & $151(32.0)$ & $136(34.1)$ & 152(38.2) & 0.005 \\
\hline Educational level, n (\%) & & & & & $<0.001$ \\
\hline primary school and less than & $71(21.8)$ & 71(15.0) & $40(10.00)$ & $34(8.5)$ & \\
\hline junior middle and high school & $154(47.4)$ & $228(48.3)$ & 197(49.4) & $209(52.5)$ & \\
\hline junior college or above & $100(30.8)$ & $173(36.7)$ & $162(40.6)$ & 155(38.9) & \\
\hline Income (yuan/month), n (\%) & & & & & $<0.001$ \\
\hline$<2000$ & $52(16.0)$ & $36(7.6)$ & $28(7.0)$ & $24(6.0)$ & \\
\hline $2000-3000$ & $131(40.3)$ & $167(35.4)$ & $121(30.3)$ & $120(30.2)$ & \\
\hline$>3000$ & 142(43.7) & $269(57.0)$ & $250(62.7)$ & $254(63.8)$ & \\
\hline Marital status, n (\%) & & & & & $<0.001$ \\
\hline Married & $312(96.0)$ & $469(99.4)$ & $391(98.0)$ & 397(99.7) & \\
\hline Single or other & $13(4.0)$ & $3(0.6)$ & $8(2.0)$ & $1(0.3)$ & \\
\hline Smoking status, n (\%) & & & & & $<0.001$ \\
\hline Never & $264(81.2)$ & $382(80.9)$ & 283(70.9) & $248(62.3)$ & \\
\hline Former & $17(5.2)$ & $20(4.2)$ & $24(6.0)$ & $34(8.55)$ & \\
\hline Current & $44(13.5)$ & $70(14.8)$ & $92(23.11)$ & 116(29.1) & \\
\hline Tea intake status, n (\%) & & & & & $<0.001$ \\
\hline No & $168(51.7)$ & 218(46.2) & $147(36.8)$ & $136(34.2)$ & \\
\hline Yes & 157(48.3) & 254(53.8) & 252(63.2) & $262(65.8)$ & \\
\hline $\mathrm{BMI}\left(\mathrm{kg} / \mathrm{m}^{2}\right), M(I Q R)$ & $\begin{array}{l}22.72(21.06- \\
24.98)\end{array}$ & $23.60(21.64-25.40)$ & $23.41(21.48-25.64)$ & $\begin{array}{l}24.10(22.49- \\
26.09)\end{array}$ & $<0.001$ \\
\hline Waist circumference (cm), $M(I Q R)$ & 83(76-89) & $83(77-90)$ & $85(78-91)$ & $86(80-92)$ & $<0.001$ \\
\hline Hip circumference $(\mathrm{cm}), M(I Q R)$ & $94(91-98)$ & $95(91-99)$ & $96(92-99)$ & 97(93-101) & $<0.001$ \\
\hline $\mathrm{SBP}(\mathrm{mmHg}), M(I Q R)$ & $124(112-140)$ & $120(110-132)$ & $120(110-131)$ & $122(112-134)$ & 0.124 \\
\hline $\mathrm{DBP}(\mathrm{mmHg}), M(I Q R)$ & $80(74-88)$ & $80(77-88)$ & $80(75-88)$ & $80(76-90)$ & 0.317 \\
\hline Hypertension, n (\%) & $132(40.6)$ & $170(36.0)$ & $148(37.1)$ & 157(39.4) & 0.528 \\
\hline Dyslipidemia, n (\%) & $121(37.2)$ & $165(35.0)$ & $144(36.1)$ & $162(40.7)$ & 0.345 \\
\hline Diabetes, n (\%) & $66(20.3)$ & $96(20.3)$ & 77(19.3) & $74(18.6)$ & 0.909 \\
\hline GGT (U/L), $M(I Q R)$ & $22(17-33)$ & $24(17-36)$ & $26(18-39)$ & $28(20-44)$ & $<0.001$ \\
\hline $\operatorname{ALT}(\mathrm{U} / \mathrm{L}), M(I Q R)$ & $19(14-25)$ & $20(15-27)$ & $21(16-29)$ & $21(16-30)$ & 0.001 \\
\hline
\end{tabular}

Page 5/16 


\begin{tabular}{|c|c|c|c|c|c|}
\hline AST (U/L), $M(I Q R)$ & $22(19-26)$ & $22(19-26)$ & $22(19-26)$ & $23(19-27)$ & 0.318 \\
\hline $\mathrm{FPG}(\mathrm{mmol} / \mathrm{L}), M(I Q R)$ & $5.30(4.99-5.80)$ & $5.28(5.01-5.83)$ & $5.28(4.95-5.71)$ & $5.30(4.98-5.76)$ & 0.708 \\
\hline $\mathrm{TC}(\mathrm{mmol} / \mathrm{L}), M(I Q R)$ & $5.20(4.64-5.71)$ & $5.28(4.70-5.80)$ & $5.26(4.75-5.97)$ & $5.20(4.75-5.91)$ & 0.328 \\
\hline $\mathrm{TG}(\mathrm{mmol} / \mathrm{L}), M(I Q R)$ & $1.35(0.97-1.93)$ & 1.44(1.03-1.97) & $1.43(1.04-2.01)$ & $1.47(1.05-2.07)$ & 0.354 \\
\hline LDL (mmol/L), $M(I Q R)$ & $3.31(2.70-3.83)$ & $3.35(2.81-3.89)$ & $3.31(2.81-3.96)$ & $3.32(2.70-3.80)$ & 0.610 \\
\hline $\mathrm{HDL}(\mathrm{mmol} / \mathrm{L}), M(I Q R)$ & $1.32(1.15-1.46)$ & $1.32(1.14-1.46)$ & $1.32(1.14-1.46)$ & $1.29(1.11-1.44)$ & 0.124 \\
\hline $\begin{array}{l}\text { Physical activity ( hours/week), } M \\
(I Q R)\end{array}$ & $10.5(4.5-17.5)$ & $9.5(4.0-15.3)$ & $8.9(4.1-15.0)$ & 7.0(3.5-15.6) & 0.051 \\
\hline
\end{tabular}

BMI, body mass index; SBP, systolic blood pressure; DBP, diastolic blood pressure; GGT, y-glutamyl transferase; ALT, alanine aminotransferase; AST, aspartate aminotransferase; FPG, fasting plasma-glucose; TC, total cholesterol; TG, total triglyceride; LDL, lowdensity lipoprotein; HDL, high-density lipoprotein; NAFLD, non-alcoholic fatty liver disease; $M(I Q R)=$ Median (Interquartile range); $Q=$ quartile.

\section{Meat subtypes consumptions and NAFLD}

As presented in Table 2, after propensity score weighting, red meat intake was positively related with the risk of NAFLD (OR per $50 \mathrm{~g} / \mathrm{day}$ of red meat: 1.128, 95\% Cl: 1.005-1.266). After further adjustment for smoking status, tea intake status, weekly hours of physical activity and the presence of hypertension, dyslipidemia and diabetes, the association with NAFLD remained significant $\left(O R_{\text {adjusted }}=1.143\right)$. High

consumptions of red $\left(Q_{2}\right.$ and $\left.Q_{4}\right)$ was significantly relevant to higher odds for NAFLD ( $O R_{\text {adjusted }}=1.948$ and 1.716 , respectively), adjusting for those potential confounders.

Table 2. Propensity score weighted univariable and multivariable analysis of associations between meat subtypes consumptions and NAFLD 


\begin{tabular}{|c|c|c|}
\hline Variable & Crude $O R(95 \% C l)$ & Adjusted $O R(95 \% C /)^{\mathrm{a}}$ \\
\hline \multicolumn{3}{|l|}{ Red meat } \\
\hline \multicolumn{3}{|l|}{ Continuous variable } \\
\hline Per $50 \mathrm{~g} /$ day increase & $1.128(1.005-1.266)$ & $1.143(1.010-1.294)$ \\
\hline \multicolumn{3}{|l|}{ Categorical variables } \\
\hline$Q_{1}(<28.44 \mathrm{~g} /$ day $)$ & 1(Reference) & 1(Reference) \\
\hline$Q_{2}(28.44-49.74 \mathrm{~g} /$ day $)$ & $1.901(1.386-2.609)$ & $1.948(1.399-2.714)$ \\
\hline$Q_{3}(49.75-71.00 \mathrm{~g} /$ day $)$ & $1.221(0.872-1.710)$ & $1.190(0.833-1.698)$ \\
\hline$Q_{4}(>71.00 \mathrm{~g} /$ day $)$ & $1.659(1.199-2.295)$ & $1.716(1.214-2.424)$ \\
\hline$P$ for trend & 0.043 & 0.043 \\
\hline \multicolumn{3}{|l|}{ Processed meat } \\
\hline \multicolumn{3}{|l|}{ Continuous variable } \\
\hline Per $50 \mathrm{~g} /$ day increase & $1.003(0.806-1.247)$ & $0.965(0.766-1.216)$ \\
\hline \multicolumn{3}{|l|}{ Categorical variables } \\
\hline$Q_{1}(<2.26 \mathrm{~g} /$ day $)$ & 1(Reference) & 1(Reference) \\
\hline$Q_{2}(2.26-4.61 \mathrm{~g} / \mathrm{day})$ & $1.159(0.828-1.622)$ & $1.146(0.806-1.629)$ \\
\hline$Q_{3}(4.62-6.59 \mathrm{~g} /$ day $)$ & $1.521(1.104-2.096)$ & $1.389(0.992-1.946)$ \\
\hline$Q_{4}(>6.59 \mathrm{~g} / \mathrm{day})$ & $1.376(0.986-1.921)$ & $1.335(0.938-1.901)$ \\
\hline$P$ for trend & 0.020 & 0.059 \\
\hline \multicolumn{3}{|l|}{ Poultry } \\
\hline \multicolumn{3}{|l|}{ Continuous variable } \\
\hline Per $50 \mathrm{~g} /$ day increase & $0.974(0.666-1.424)$ & $1.021(0.681-1.531)$ \\
\hline \multicolumn{3}{|l|}{ Categorical variables } \\
\hline$Q_{1}(<7.00 \mathrm{~g} /$ day $)$ & 1(Reference) & 1(Reference) \\
\hline$Q_{2}(7.00-9.00 \mathrm{~g} / \mathrm{day})$ & $1.145(0.829-1.582)$ & $1.131(0.805-1.589)$ \\
\hline$Q_{3}(9.01-14.00 \mathrm{~g} /$ day $)$ & $1.086(0.806-1.462)$ & $0.998(0.730-1.364)$ \\
\hline$Q_{4}(>14.00 \mathrm{~g} /$ day $)$ & $1.052(0.774-1.431)$ & $1.078(0.779-1.493)$ \\
\hline$P$ for trend & 0.753 & 0.801 \\
\hline \multicolumn{3}{|l|}{ Fish } \\
\hline \multicolumn{3}{|l|}{ Continuous variable } \\
\hline Per 50 g/day increase & $0.967(0.868-1.077)$ & $0.989(0.883-1.108)$ \\
\hline \multicolumn{3}{|l|}{ Categorical variables } \\
\hline$Q_{1}(<11.01 \mathrm{~g} /$ day $)$ & 1(Reference) & 1(Reference) \\
\hline$Q_{2}(11.01-25.15 \mathrm{~g} /$ day $)$ & $0.865(0.632-1.184)$ & $0.870(0.625-1.211)$ \\
\hline$Q_{3}(25.16-50.42 \mathrm{~g} /$ day $)$ & $1.007(0.735-1.379)$ & $1.013(0.727-1.411)$ \\
\hline$Q_{4}(>50.42 \mathrm{~g} /$ day $)$ & $0.922(0.669-1.271)$ & $0.943(0.671-1.324)$ \\
\hline
\end{tabular}




\begin{tabular}{|cll|}
\hline Variable & Crude $O R(95 \% C l)$ & Adjusted $O R(95 \% C)^{\text {a }}$ \\
\hline$P$ for trend & 0.870 & 0.976 \\
\hline
\end{tabular}

NAFLD, non-alcoholic fatty liver diseases.

a Adjusted by smoking status, tea intake status, weekly hours of physical activity and the presence of hypertension, dyslipidemia and diabetes. $Q=$ quartile.

The restricted cubic splines analyses were applied to explain the exposure-response association between meat subtypes intakes and the risk of NAFLD (Fig. 1). The ORs of NAFLD increased with red meat and poultry consumptions, but decreased with fish intake (Fig. 1A, 1C and 1D). Moreover, the trend for OR of processed meat intake was not found (Fig. 1B).

\section{Stratified analyses}

The positive association between meat intake and NAFLD was consistent across strata of age, sex, smoking status, tea intake status, BMI, weekly hours of physical activity, and the presence of hypertension, dyslipidemia and diabetes. Significant associations between red meat intake and the risk of NAFLD were not only found in males, but also existed in people whose BMI $\geq 24 \mathrm{~kg} / \mathrm{m}^{2}$, tea-drinkers, people with hypertension and people without dyslipidemia. Adjusted ORs were estimated to be 1.177, 1.195, 1.229, 1.304 and 1.203, respectively. Moreover, fish intake was found positively relevant to NAFLD in people with diabetes (OR $\left.R_{\text {adjusted }}=1.447\right)$. Nonetheless, no significant interactions of meat subtypes intakes and potential confounders were identified (Table 3).

Table 3. Stratified analysis of association between meat subtypes intakes and NAFLD risk 


\begin{tabular}{|c|c|c|c|c|c|c|c|c|}
\hline \multirow{2}{*}{ Subgroup } & \multicolumn{2}{|c|}{ Red meat (per $50 \mathrm{~g} /$ day) } & \multicolumn{2}{|c|}{$\begin{array}{l}\text { Processed meat (per } 50 \\
\text { g/day) }\end{array}$} & \multicolumn{2}{|c|}{ Poultry (per 50 g/day) } & \multicolumn{2}{|c|}{ Fish (per 50 g/day) } \\
\hline & $O R(95 \% C l)$ & $\begin{array}{l}P \text { for } \\
\text { interaction }\end{array}$ & $O R(95 \% C l)$ & $\begin{array}{l}P \text { for } \\
\text { interaction }\end{array}$ & $O R(95 \% C l)$ & $\begin{array}{l}P \text { for } \\
\text { interaction }\end{array}$ & $O R(95 \% C l)$ & $\begin{array}{l}P \text { for } \\
\text { interaction }\end{array}$ \\
\hline Gender & & 0.259 & & 0.759 & & 0.565 & & 0.844 \\
\hline Male & $\begin{array}{l}1.177(1.013- \\
1.368)\end{array}$ & & $\begin{array}{l}0.991(0.798- \\
1.231)\end{array}$ & & $\begin{array}{l}1.156(0.737- \\
1.838)\end{array}$ & & $\begin{array}{l}0.962(0.820- \\
1.128)\end{array}$ & \\
\hline Female & $\begin{array}{l}1.097(0.883- \\
1.364)\end{array}$ & & $\begin{array}{l}1.165(0.619- \\
2.193)\end{array}$ & & $\begin{array}{l}0.896(0.397- \\
2.018)\end{array}$ & & $\begin{array}{l}1.009(0.857- \\
1.188)\end{array}$ & \\
\hline Age & & 0.503 & & 0.208 & & 0.121 & & 0.220 \\
\hline$<60$ years & $\begin{array}{l}1.112(0.969- \\
1.276)\end{array}$ & & $\begin{array}{l}1.004(0.812- \\
1.243)\end{array}$ & & $\begin{array}{l}0.990(0.649- \\
1.510)\end{array}$ & & $\begin{array}{l}0.956(0.841- \\
1.087)\end{array}$ & \\
\hline${ }^{3} 60$ years & $\begin{array}{l}1.255(0.956- \\
1.649)\end{array}$ & & $\begin{array}{l}3.397(0.657- \\
17.567)\end{array}$ & & $\begin{array}{l}2.134(0.686- \\
6.636)\end{array}$ & & $\begin{array}{l}1.201(0.870- \\
1.657)\end{array}$ & \\
\hline BMI & & 0.543 & & 0.346 & & 0.391 & & 0.947 \\
\hline$<24 \mathrm{~kg} / \mathrm{m}^{2}$ & $\begin{array}{l}1.141(0.906- \\
1.438)\end{array}$ & & $\begin{array}{l}0.856(0.508- \\
1.441)\end{array}$ & & $\begin{array}{l}1.124(0.624- \\
2.024)\end{array}$ & & $\begin{array}{l}1.025(0.873- \\
1.205)\end{array}$ & \\
\hline${ }^{3} 24 \mathrm{~kg} / \mathrm{m}^{2}$ & $\begin{array}{l}1.195(1.029- \\
1.387)\end{array}$ & & $\begin{array}{l}1.196(0.789- \\
1.811)\end{array}$ & & $\begin{array}{l}1.542(0.880- \\
2.704)\end{array}$ & & $\begin{array}{l}1.002(0.855- \\
1.175)\end{array}$ & \\
\hline Smoking status & & 0.425 & & 0.811 & & 0.746 & & 0.732 \\
\hline Never & $\begin{array}{l}1.079(0.916- \\
1.271)\end{array}$ & & $\begin{array}{l}1.195(0.685- \\
2.082)\end{array}$ & & $\begin{array}{l}1.332(0.747- \\
2.376)\end{array}$ & & $\begin{array}{l}1.018(0.888- \\
1.168)\end{array}$ & \\
\hline Former & $\begin{array}{l}1.560(0.908- \\
2.680)\end{array}$ & & $\begin{array}{l}1.422(0.052- \\
39.060)\end{array}$ & & $\begin{array}{l}1.246(0.228- \\
6.818)\end{array}$ & & $\begin{array}{l}1.047(0.697- \\
1.573)\end{array}$ & \\
\hline Current & $\begin{array}{l}1.176(0.959- \\
1.442)\end{array}$ & & $\begin{array}{l}1.006(0.798- \\
1.269)\end{array}$ & & $\begin{array}{l}0.936(0.535- \\
1.638)\end{array}$ & & $\begin{array}{l}0.919(0.726- \\
1.164)\end{array}$ & \\
\hline $\begin{array}{l}\text { Tea intake } \\
\text { status }\end{array}$ & & 0.095 & & 0.842 & & 0.201 & & 0.745 \\
\hline No & $\begin{array}{l}1.014(0.832- \\
1.235)\end{array}$ & & $\begin{array}{l}1.006(0.808- \\
1.253)\end{array}$ & & $\begin{array}{l}0.822(0.430- \\
1.570)\end{array}$ & & $\begin{array}{l}0.965(0.726- \\
1.281)\end{array}$ & \\
\hline Yes & $\begin{array}{l}1.229(1.048- \\
1.442)\end{array}$ & & $\begin{array}{l}1.037(0.499- \\
2.155)\end{array}$ & & $\begin{array}{l}1.413(0.838- \\
2.385)\end{array}$ & & $\begin{array}{l}0.994(0.875- \\
1.131)\end{array}$ & \\
\hline Hypertension & & 0.166 & & 0.195 & & 0.363 & & 0.094 \\
\hline No & $\begin{array}{l}1.037(0.884- \\
1.216)\end{array}$ & & $\begin{array}{l}1.269(0.870- \\
1.852)\end{array}$ & & $\begin{array}{l}0.935(0.568- \\
1.537)\end{array}$ & & $\begin{array}{l}0.905(0.760- \\
1.079)\end{array}$ & \\
\hline Yes & $\begin{array}{l}1.304(1.063- \\
1.598)\end{array}$ & & $\begin{array}{l}0.766(0.389- \\
1.508)\end{array}$ & & $\begin{array}{l}1.440(0.752- \\
2.757)\end{array}$ & & $\begin{array}{l}1.138(0.945- \\
1.369)\end{array}$ & \\
\hline Dyslipidemia & & 0.245 & & 0.118 & & 0.607 & & 0.709 \\
\hline No & $\begin{array}{l}1.203(1.017- \\
1.424)\end{array}$ & & $\begin{array}{l}1.686(0.806- \\
3.526)\end{array}$ & & $\begin{array}{l}1.208(0.709- \\
2.056)\end{array}$ & & $\begin{array}{l}0.999(0.860- \\
1.159)\end{array}$ & \\
\hline Yes & $\begin{array}{l}1.072(0.898- \\
1.278)\end{array}$ & & $\begin{array}{l}0.958(0.759- \\
1.211)\end{array}$ & & $\begin{array}{l}1.037(0.594- \\
1.812)\end{array}$ & & $\begin{array}{l}0.975(0.811- \\
1.172)\end{array}$ & \\
\hline Diabetes & & 0.973 & & 0.852 & & 0.112 & & 0.110 \\
\hline No & $\begin{array}{l}1.129(0.988- \\
1.290)\end{array}$ & & $\begin{array}{l}1.001(0.810- \\
1.237)\end{array}$ & & $\begin{array}{l}0.978(0.631- \\
1.518)\end{array}$ & & $\begin{array}{l}0.936(0.813- \\
1.076)\end{array}$ & \\
\hline Yes & $\begin{array}{l}1.272(0.923- \\
1.753)\end{array}$ & & $\begin{array}{l}1.206(0.357- \\
4.073)\end{array}$ & & $\begin{array}{l}2.816(0.990- \\
8.008)\end{array}$ & & $\begin{array}{l}1.447(1.017- \\
2.059)\end{array}$ & \\
\hline $\begin{array}{l}\text { Physical } \\
\text { activity }\end{array}$ & & 0.745 & & 0.730 & & 0.653 & & 0.736 \\
\hline$<9.0$ & 1.135(0.949- & & $0.800(0.346-$ & age $9 / 16$ & $1.211(0.716-$ & & 1.001(0.828- & \\
\hline
\end{tabular}




\begin{tabular}{|lllll|} 
hours/week & $1.356)$ & $1.851)$ & $2.046)$ & $1.211)$ \\
\hline 39.0 & $1.134(0.957-$ & $1.024(0.829-$ & $1.070(0.600-$ & $0.986(0.851-$ \\
hours/week & $1.344)$ & $1.266)$ & $1.908)$ & $1.142)$ \\
\hline
\end{tabular}

NAFLD, non-alcoholic fatty liver diseases; BMI, body mass index.

* Adjusted for age, gender, BMI, smoking status, tea intake status, weekly hours of physical activity and the presence of hypertension, dyslipidemia and diabetes.

\section{Sensitivity analyses}

Results from unweighted analysis were similar to those from propensity score-weighted analysis. Compared with the lowest quartile of meat intake, high intakes of red meat $\left(Q_{2}\right.$ and $\left.Q_{4}\right)$ were significantly associated with NAFLD risk without inverse probability of treatment weighting $\left(O R_{\text {adjusted }}=1.484\right.$ and 1.558 , respectively) (see Additional file 1$)$. Nonetheless, positive associations of red meat $\left(Q_{4}\right)$ and processed meat $\left(Q_{3}\right)$ consumptions with NAFLD risk were observed on propensity score matching analysis, with adjusted $O R$ of 1.673 and 1.800 , respectively (see Additional file 2).

\section{Meat subtypes intakes and liver-related biochemical indexes}

Associations between meat subtypes intakes and liver-related biochemical indexes were shown in Fig. 2. Red meat intake was positively correlated with levels of GGT, ALT, AST and total triglyceride (TG), but inversely with high-density lipoprotein cholesterol (HDL-C) (Spearman test correlation coefficient $=0.176,0.128,0.060,0.085$ and -0.074 , respectively). Processed meat intake was significantly associated with GGT, ALT, TG and HDL-C (Spearman test correlation coefficient $=0.141,0.115,0.081$ and -0.067 , respectively). Similarly, significant associations were observed between poultry intake and GGT, ALT, TG and HDL-C (Spearman test correlation coefficient $=0.111,0.104,0.085$ and -0.066 , respectively). In addition, GGT was also found to be significantly related to the consumption of fish (Spearman test correlation coefficient $=0.063$ ).

\section{Discussion}

In this cross-sectional study, we observed that NAFLD was associated with higher intake levels of red meat. Significant associations of serum levels of GGT, ALT, AST, TG and HDL-C with meat subtypes intakes were found as well. Additionally, no significant interactions between meat consumptions and potential confounders for NAFLD were detected.

Significant associations between high meat consumptions and NAFLD were demonstrated in a few studies [7, 9, 10,26]. Our results are in accordance with the previous studies, indicating a positive association between high red meat intake and NAFLD. Two cross-sectional studies have presented that red meat was significantly correlated with NAFLD [7, 10]. In another cross-sectional study, high intakes of total meat, especially red meat and/or processed meat were positively linked to NAFLD and insulin resistance, while processed meat alone was only relevant to insulin resistance. This is mainly due to a relatively low level of processed meat consumption in their research set [9]. In addition, a nested case-control study also showed that high consumptions of red meat, processed red meat and poultry were positively associated with NAFLD [26]. Due to the better accuracy of the continuous ORs [27], the dose-response analysis we have employed can better measure the overall trends of the ORs for meat intakes. The $95 \% \mathrm{C} /$ for red meat intake beyond $200 \mathrm{~g} /$ day was slightly wider, because that less than $2 \%$ of participants had red meat intake $>200 \mathrm{~g} /$ day, that was, the tendency of red meat intake within the range $0 \sim 200 \mathrm{~g} / \mathrm{day}$ was relatively reliable and stable.

There are several plausible mechanisms by which meat intake is related to NAFLD. NAFLD was reported to be closely linked to hepatic insulin resistance, which had strong correlation with liver biomarkers such as ALT, AST and GGT [28]. GGT and ALT had been considered as biomarkers of hepatic fat accumulation, which can lead to hepatic insulin resistance and increase the contribution of gluconeogenesis to total endogenous glucose production [29]. A cross-sectional study of 2198 European reported a significant positive association between red meat and GGT. As GGT is also a potential nonspecific marker of oxidative stress, the author suggested that oxidative stress may plays a vital role underlying the development of chronic diseases with red meat intake [30]. Another cross-sectional study indicated that TG/HDL-C was independently relevant to the risk of NAFLD. The author attributed this result to insulin resistance [31]. Positive associations of serum levels of GGT, ALT and TG with red meat intakes were found in this study. Inversely, serum HDL-C concentration was negatively relevant to red meat and processed meat intakes. Hence, it's plausible that increased hepatic lipid accumulation and insulin resistance play a substantial role in the relationship of meat intakes with the development of NAFLD. In addition, a study by Avila et al. found that red meat was positively relevant to serum ferritin [32], which can increase the risk of NAFLD [33, 34]. Fried food will produce some hazardous chemicals, such as advanced glycation end products and trans fatty acids [35,36], which were reported to play a critical role in NAFLD [37, 38]. 
Significant associations were found in several subgroups. A positive association was observed between red meat and NAFLD in males, perhaps it is because males had a higher meat intakes and a higher prevalence of fatty liver than females [39]. Moreover, several studies had found significant associations of high meat intakes with obesity, type 2 diabetes and hypertension [14, 17, 40], which were considered as risk factors in the development of NAFLD [19, 41, 42]. In our study, positive association of red meat intake with NAFLD was found in people with $B M I \geq 24 \mathrm{~kg} / \mathrm{m}^{2}$ and people with hypertension.

This study, however, had several limitations. Firstly, because of the cross-sectional study design, casual inference was not allowed. Secondly, measurement error was unavoidable for self-reported diet and other data. Nevertheless, since all participants and researchers in this study were blinded to the results of abdominal ultrasonography and blood test, a reporting bias without differences is likely only to attenuate our observed association. Thirdly, since the study subjects were middle-aged and elders, it should be cautious in generalizing our findings to the wider population. Lastly, although a comprehensive set of confounders were considered, as an observational study, the presence of unmeasured confounders is possible.

\title{
Conclusions
}

In conclusion, positive relationship between high consumptions of red meat and the risk of NAFLD was observed. In addition, serum levels of liver-related biomarkers were significantly relevant to red meat intake. Our findings suggested that reduction of meat consumption may decrease the risk of NAFLD.

\section{Abbreviations}

\author{
NAFLD \\ Non-alcoholic fatty liver disease \\ BMI \\ Body mass index \\ GGT \\ Y-glutamyl transferase \\ AST \\ Aspartate aminotransferase \\ ALT \\ Alanine transaminase \\ TG \\ Total triglyceride \\ HDL-C \\ High-density lipoprotein cholesterol \\ IQR \\ Interquartile range \\ IPTW \\ Inverse probability of treatment weighting analysis
}

\section{Declarations}

\section{Ethics approval and consent to participate}

The current study was carried out in compliance with the Declaration of Helsinki, and the Ethics Committee of Fujian Medical University approved the study protocol (ethics number 2014096). All subjects provided their informed consent prior to participating in this study.

\section{Consent for publication}

Not applicable.

\section{Availability of data and materials}

The datasets used can be available from the corresponding author on reasonable request. 


\section{Competing interests}

All the authors declare that there are no financial or other relationships that might lead to a conflict of interest of the present article.

\section{Authors' contributions}

PHW and XXX are joint first authors. PXE, HZJ and ZYD designed the study. PHW, PXT, ZJ and CXL collected the data. PHW, XXX and PXT analysed the data. HZJ, ZJ, ZYD and CXL contributed to the interpretation of results. PHW, XXX and PXE wrote and reviewed the manuscript. All authors have discussed the results and commented on the manuscript.

\section{Fundings}

This work was supported by the National Natural Science Foundation of China (grant no. 81473047), the Joint Funds for the Innovation of Science and Technology, Fujian Province (grant no. 2017Y9104), the National Natural Science Foundation of Fujian Province (grant no. 2019J01316), and the Fujian Medical University talent research funding (grant no: XRCZX2019031).

\section{Acknowledgements}

The authors would like to express their gratitude to all participants for their cooperation and to all staffs for recruiting subjects and their technical assistance.

\section{Authors' information}

Hewei Peng and Xiaoxu Xie contributed equally to this work

\section{Afflictions}

Department of Epidemiology and Health Statistics, Fujian Provincial Key Laboratory of Environment Factors and Cancer, School of Public Health, Fujian Medical University, Fuzhou, PR China

Hewei Peng, Xiaoxu Xie, Xinting Pan, Jing Zheng, Yidan Zeng, Xiaoling Cai, Zhijian Hu \& Xian-E Peng

Key Laboratory of Ministry of Education for Gastrointestinal Cancer, Fujian Medical University, Fuzhou, PR China

Xian-E Peng

\section{Contact information}

phw@fjmu.edu.cn, xiexiaoxu@aliyun.com, panxinting@fjmu.edu.cn, fjmuzj@fjmu.edu.cn, zengyidan@126.com, cxl15059154060@fjmu.edu.cn, huzhijian@fjmu.edu.cn \& fmuxe@163.com

\section{Corresponding author}

Correspondence to Xian-E Peng.

\section{References}

1. Younossi ZM, Koenig AB, Abdelatif D, Fazel Y, Henry L, Wymer M. Global epidemiology of nonalcoholic fatty liver disease-Meta-analytic assessment of prevalence, incidence, and outcomes. Hepatology. 2016;64:73-84. doi:10.1002/hep.28431.

2. Friedman SL, Neuschwander-Tetri BA, Rinella M, Sanyal AJ. Mechanisms of NAFLD development and therapeutic strategies. Nat Med. 2018;24:908-22. doi:10.1038/s41591-018-0104-9.

3. Anstee QM, Targher G, Day CP. Progression of NAFLD to diabetes mellitus, cardiovascular disease or cirrhosis. Nat Rev Gastroenterol Hepatol. 2013;10:330-44. doi:10.1038/nrgastro.2013.41.

4. Gaggini M, Morelli M, Buzzigoli E, DeFronzo RA, Bugianesi E, Gastaldelli A. Non-alcoholic fatty liver disease (NAFLD) and its connection with insulin resistance, dyslipidemia, atherosclerosis and coronary heart disease. Nutrients. 2013;5:1544-60. doi:10.3390/nu5051544. 
5. Romero-Gómez M, Zelber-Sagi S, Trenell M. Treatment of NAFLD with diet, physical activity and exercise. J Hepatol. 2017;67:829-46. doi:10.1016/j.jhep.2017.05.016.

6. $10.3390 /$ nu 9080800

Eslamparast T, Tandon P, Raman M. Dietary Composition Independent of Weight Loss in the Management of Non-Alcoholic Fatty Liver Disease. Nutrients 2017. doi:10.3390/nu9080800.

7. Zelber-Sagi S, Nitzan-Kaluski D, Goldsmith R, Webb M, Blendis L, Halpern Z, Oren R. Long term nutritional intake and the risk for nonalcoholic fatty liver disease (NAFLD): a population based study. J Hepatol. 2007;47:711-7. doi:10.1016/j.jhep.2007.06.020.

8. Oddy WH, Herbison CE, Jacoby P, Ambrosini GL, O'Sullivan TA, Ayonrinde OT, et al. The Western dietary pattern is prospectively associated with nonalcoholic fatty liver disease in adolescence. Am J Gastroenterol. 2013;108:778-85. doi:10.1038/ajg.2013.95.

9. Zelber-Sagi S, Ivancovsky-Wajcman D, Fliss Isakov N, Webb M, Orenstein D, Shibolet O, Kariv R. High red and processed meat consumption is associated with non-alcoholic fatty liver disease and insulin resistance. J Hepatol. 2018;68:1239-46. doi:10.1016/j.jhep.2018.01.015.

10. Liu X, Peng Y, Chen S, Sun Q. An observational study on the association between major dietary patterns and non-alcoholic fatty liver disease in Chinese adolescents. Med (Baltim). 2018;97:e0576. doi:10.1097/MD.0000000000010576.

11. Godfray HCJ, Aveyard P, Garnett T, Hall JW, Key TJ, Lorimer J, et al. Meat consumption, health, and the environment. Science. 2018. doi:10.1126/science.aam5324.

12. Ekmekcioglu C, Wallner P, Kundi M, Weisz U, Haas W, Hutter H-P. Red meat, diseases, and healthy alternatives: A critical review. Crit Rev Food Sci Nutr. 2018;58:247-61. doi:10.1080/10408398.2016.1158148.

13. He Y, Yang X, Xia J, Zhao L, Yang Y. Consumption of meat and dairy products in China: a review. Proc Nutr Soc. 2016;75:385-91. doi:10.1017/S0029665116000641.

14. Micha R, Michas G, Mozaffarian D. Unprocessed red and processed meats and risk of coronary artery disease and type 2 diabetes-an updated review of the evidence. Curr Atheroscler Rep. 2012;14:515-24. doi:10.1007/s11883-012-0282-8.

15. Zhong VW, van Horn L, Greenland P, Carnethon MR, Ning H, Wilkins JT, et al. Associations of Processed Meat, Unprocessed Red Meat, Poultry, or Fish Intake With Incident Cardiovascular Disease and All-Cause Mortality. JAMA Intern Med. 2020;180:503. doi:10.1001/jamainternmed.2019.6969.

16. Freedman ND, Cross AJ, McGlynn KA, Abnet CC, Park Y, Hollenbeck AR, et al. Association of meat and fat intake with liver disease and hepatocellular carcinoma in the NIH-AARP cohort. J Natl Cancer Inst. 2010;102:1354-65. doi:10.1093/jnci/djq301.

17. Schwingshackl L, Schwedhelm C, Hoffmann G, Knüppel S, Iqbal K, Andriolo V, et al. Food Groups and Risk of Hypertension: A Systematic Review and Dose-Response Meta-Analysis of Prospective Studies. Adv Nutr. 2017;8:793-803. doi:10.3945/an.117.017178.

18. Francque SM, van der Graaff D, Kwanten WJ. Non-alcoholic fatty liver disease and cardiovascular risk: Pathophysiological mechanisms and implications. J Hepatol. 2016;65:425-43. doi:10.1016/j.jhep.2016.04.005.

19. Lonardo A, Nascimbeni F, Mantovani A, Targher G. Hypertension, diabetes, atherosclerosis and NASH: Cause or consequence? J Hepatol. 2018;68:335-52. doi:10.1016/j.jhep.2017.09.021.

20. Marchisello S, Di Pino A, Scicali R, Urbano F, Piro S, Purrello F, Rabuazzo AM. Pathophysiological, Molecular and Therapeutic Issues of Nonalcoholic Fatty Liver Disease: An Overview. Int J Mol Sci. 2019. doi:10.3390/ijms20081948.

21. Ndrepepa G, Colleran R, Kastrati A. Gamma-glutamyl transferase and the risk of atherosclerosis and coronary heart disease. Clin Chim Acta. 2018;476:130-8. doi:10.1016/j.cca.2017.11.026.

22. Targher G, Byrne CD. Circulating Markers of Liver Function and Cardiovascular Disease Risk. Arterioscler Thromb Vasc Biol. 2015;35:2290-6. doi:10.1161/ATVBAHA.115.305235.

23. Zeng M de, Fan JG, Lu LG, Li YM, Chen CW, Wang BY, Mao YM. Guidelines for the diagnosis and treatment of nonalcoholic fatty liver diseases. J Dig Dis. 2008;9:108-12. doi:10.1111/j.1751-2980.2008.00331.x.

24. Kopin L, Lowenstein C. Dyslipidemia. Ann Intern Med. 2017;167:ITC81-96. doi:10.7326/AITC201712050.

25. Grool AM, Aglipay M, Momoli F, Meehan WP, Freedman SB, Yeates KO, et al. Association Between Early Participation in Physical Activity Following Acute Concussion and Persistent Postconcussive Symptoms in Children and Adolescents. JAMA. 2016;316:2504-14. doi:10.1001/jama.2016.17396.

26. $10.1002 /$ hep.30967

Noureddin M, Zelber-Sagi S, Wilkens LR, Porcel J, Boushey CJ, Le Marchand L, et al. Diet Associations With Nonalcoholic Fatty Liver Disease in an Ethnically Diverse Population: The Multiethnic Cohort. Hepatology 2019. doi:10.1002/hep.30967. 
27. Yu J, Tao Y, Dou J, Ye J, Yu Y, Jin L. The dose-response analysis between BMI and common chronic diseases in northeast China. Sci Rep. 2018;8:4228. doi:10.1038/s41598-018-22551-y.

28. Sesti G, Fiorentino TV, Hribal ML, Sciacqua A, Perticone F. Association of hepatic insulin resistance indexes to nonalcoholic fatty liver disease and related biomarkers. Nutr Metab Cardiovasc Dis. 2013;23:1182-7. doi:10.1016/j.numecd.2013.01.006.

29. Samuel VT, Liu Z-X, Qu X, Elder BD, Bilz S, Befroy D, et al. Mechanism of hepatic insulin resistance in non-alcoholic fatty liver disease. J Biol Chem. 2004;279:32345-53. doi:10.1074/jbc.M313478200.

30. Montonen J, Boeing H, Fritsche A, Schleicher E, Joost H-G, Schulze MB, et al. Consumption of red meat and whole-grain bread in relation to biomarkers of obesity, inflammation, glucose metabolism and oxidative stress. Eur J Nutr. 2013;52:337-45. doi:10.1007/s00394-012-0340-6.

31. Fan N, Peng L, Xia Z, Zhang L, Song Z, Wang Y, Peng Y. Triglycerides to high-density lipoprotein cholesterol ratio as a surrogate for nonalcoholic fatty liver disease: a cross-sectional study. Lipids Health Dis. 2019;18:39. doi:10.1186/s12944-019-0986-7.

32. Avila F, Echeverría G, Pérez D, Martinez C, Strobel P, Castillo O, et al. Serum Ferritin Is Associated with Metabolic Syndrome and Red Meat Consumption. Oxid Med Cell Longev. 2015;2015:769739. doi:10.1155/2015/769739.

33. Kowdley KV, Belt P, Wilson LA, Yeh MM, Neuschwander-Tetri BA, Chalasani N, et al. Serum ferritin is an independent predictor of histologic severity and advanced fibrosis in patients with nonalcoholic fatty liver disease. Hepatology. 2012;55:77-85. doi:10.1002/hep.24706.

34. Pan X, Chen B, Liu W, Li Y, Hu Z, Lin X, et al. Circulating Iron Levels Interaction with Central Obesity on the Risk of Nonalcoholic Fatty Liver Disease: A Case-Control Study in Southeast China. Ann Nutr Metab. 2019;74:207-14. doi:10.1159/000497228.

35. Gill V, Kumar V, Singh K, Kumar A, Kim J-J. Advanced Glycation End Products (AGEs) May Be a Striking Link Between Modern Diet and Health. Biomolecules. 2019. doi:10.3390/biom9120888.

36. Wang Y, Hui T, Zhang YW, Liu B, Wang FL, Li JK, et al. Effects of frying conditions on the formation of heterocyclic amines and trans fatty acids in grass carp (Ctenopharyngodon idellus). Food Chem. 2015;167:251-7. doi:10.1016/j.foodchem.2014.06.109.

37. Palma-Duran SA, Kontogianni MD, Vlassopoulos A, Zhao S, Margariti A, Georgoulis M, et al. Serum levels of advanced glycation endproducts (AGEs) and the decoy soluble receptor for AGEs (sRAGE) can identify non-alcoholic fatty liver disease in age-, sex-and BMImatched normo-glycemic adults. Metab Clin Exp. 2018;83:120-7. doi:10.1016/j.metabol.2018.01.023.

38. Mazidi M, Katsiki N, Mikhailidis DP, Banach M. Link between plasma trans-fatty acid and fatty liver is moderated by adiposity. Int J Cardiol. 2018;272:316-22. doi:10.1016/j.ijcard.2018.07.061.

39. Rietman A, Sluik D, Feskens EJM, Kok FJ, Mensink M. Associations between dietary factors and markers of NAFLD in a general Dutch adult population. Eur J Clin Nutr. 2018;72:117-23. doi:10.1038/ejcn.2017.148.

40. Rouhani MH, Salehi-Abargouei A, Surkan PJ, Azadbakht L. Is there a relationship between red or processed meat intake and obesity? A systematic review and meta-analysis of observational studies. Obes Rev. 2014;15:740-8. doi:10.1111/obr.12172.

41. Petrović G, Bjelaković G, Benedeto-Stojanov D, Nagorni A, Brzački V, Marković-Živković B. Obesity and metabolic syndrome as risk factors for the development of non-alcoholic fatty liver disease as diagnosed by ultrasound. Vojnosanit Pregl. 2016;73:910-20. doi:10.2298/VSP150514093P.

42. Kosmidou M, Milionis H. Diabetes mellitus and non-alcoholic fatty liver disease: the thread of Ariadne. Minerva Endocrinol. 2017;42:109-21. doi:10.23736/S0391-1977.16.02562-1.

\section{Figures}


(A

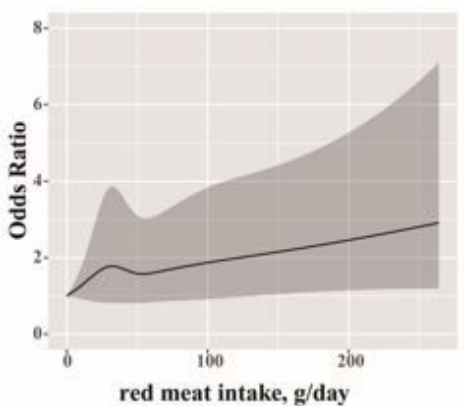

$(\mathrm{C}$

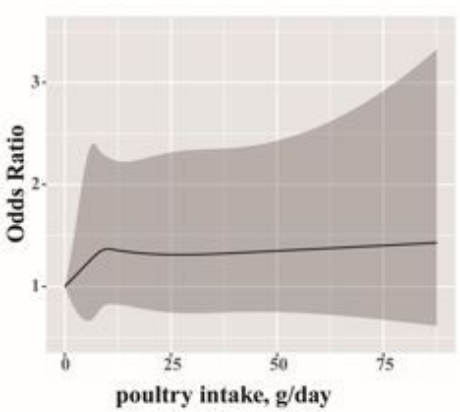

(B

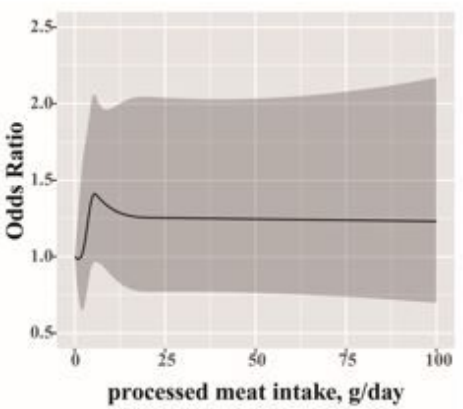

(D

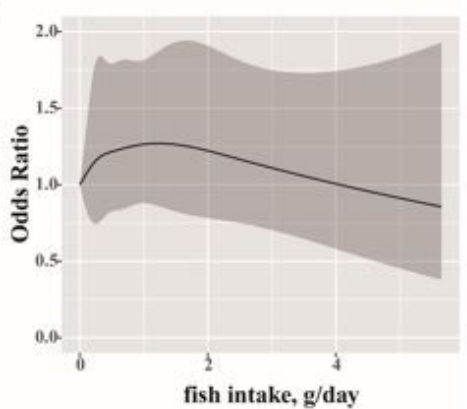

\section{Figure 1}

Restricted cubic spline model of the odds ratios of non-alcoholic fatty liver diseases (NAFLD) with intakes of (a) red meat

(Pnonlinearity=0.63), (b) processed meat (Pnonlinearity=0.24), (c) poultry (Pnonlinearity $=0.69$ ) and (d) fish (Pnonlinearity=0.55). Gray area, $95 \%$ confidence interval. Each model was adjusted for age, gender, body mass index (BMI), smoking status, tea intake status, weekly hours of physical activity and the presence of hypertension, dyslipidemia and diabetes.

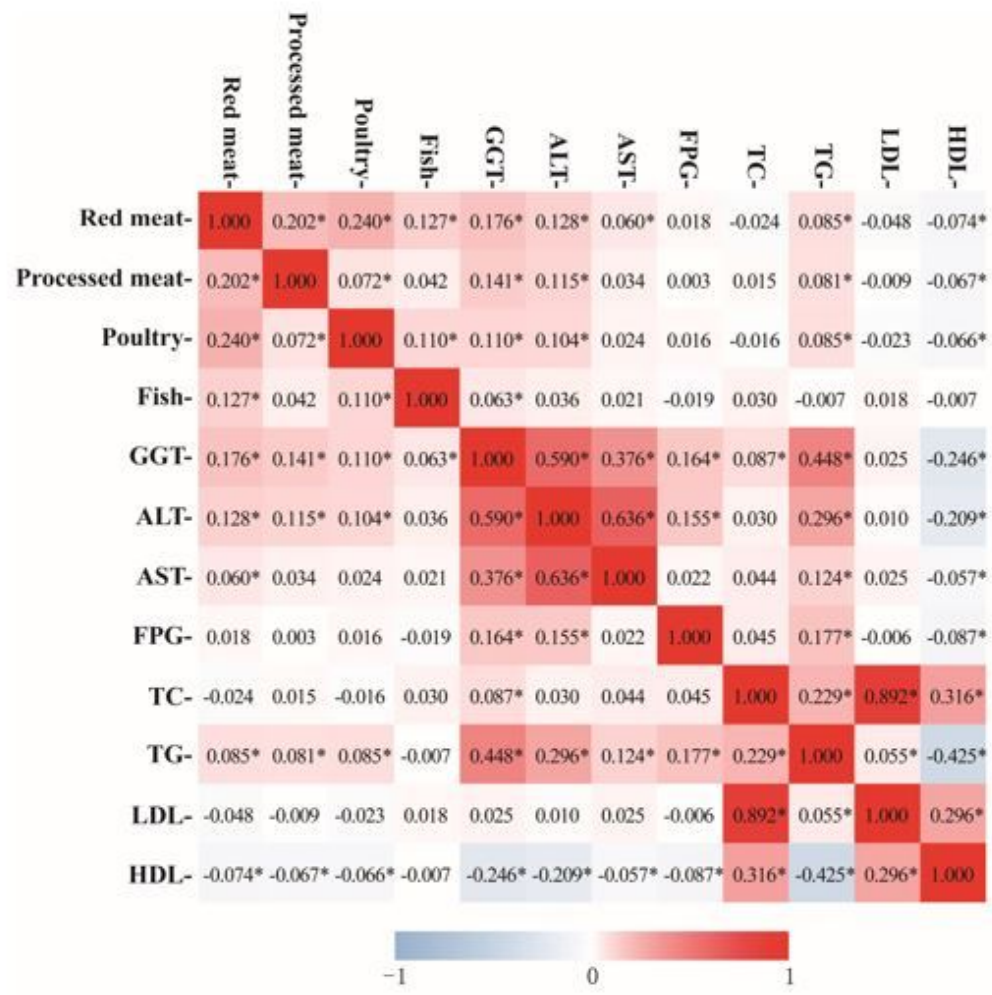

Figure 2

Heatmap of associations between meat subtypes intakes and liver-related biochemical indexes. GGT, $Y$-glutamyl transferase; ALT, alanine aminotransferase; AST, aspartate aminotransferase; FPG, fasting plasma-glucose; TC, total cholesterol; TG, total triglyceride; LDL, lowdensity lipoprotein; $\mathrm{HDL}$, high-density lipoprotein. ${ }^{*} \mathrm{P}<0.05$. 


\section{Supplementary Files}

This is a list of supplementary files associated with this preprint. Click to download.

- Additionalfile2.xls

- Additionalfile1.xls 